\title{
WI - Call for Papers Heft 3/2015
}

\section{CSCW \& Social Computing}

\section{DOI 10.1007/s11576-014-0404-8}

\section{Die Autoren}

\author{
Prof. Dr. Michael Koch ( $\varangle)$ \\ Universität der Bundeswehr \\ München \\ München \\ Deutschland \\ michael.koch@unibw.de
}

\author{
Prof. Dr. Gerhard Schwabe \\ Universität Zürich \\ Zürich \\ Schweiz \\ schwabe@ifi.uzh.ch
}

\section{Prof. Robert Briggs}

San Diego State University

San Diego

USA

robertowenbriggs@gmail.com

Online publiziert: 2014-01-22

This article is also available in English via http://www.springerlink.com and http://www.bise-journal.org: Koch M, Schwabe G, Briggs R (2013) BISE Call for Papers, Issue 3/2015. CSCW \& Social Computing. Bus Inf Syst Eng. doi: 10.1007/s12599-013-0311-3.

(C) Springer Fachmedien Wiesbaden 2014

\section{Schwerpunktthema}

Computer Supported Cooperative Work (CSCW) hat traditionell immer die Abhängigkeiten zwischen kooperierenden menschlichen Akteuren und Informationstechnologie studiert. Neue Formen von CSCW-Systemen ermöglichen nun komplett neue Weisen, Aufgaben zwischen kooperierenden Akteuren aufzuteilen - und machen dadurch den Weg für neue organisatorische Szenarien frei. Beispiele hierfür sind Crowdsourcing,
Open Innovation oder die Beteiligung von externen Experten in internen Prozessen. Diese Entwicklungen haben weitreichende Auswirkungen für Organisationsstrukturen, Management und Motivationskonzepte.

Social Computing bezieht sich auf die Unterstützung sozialer Interaktion und Sozialisierung durch Informationstechnologie. Innerhalb dieses Kontextes wird der Begriff Social Software für Werkzeuge oder Dienste genutzt, die menschliche soziale Interaktion unterstützen, erweitern oder Zusatznutzen daraus ziehen. Während beide Begriffe „Social Computing“ und „Social Software“ erst im letzten Jahrzehnt eine weitere Verbreitung gefunden haben, gehen die Kernideen dahinter viel weiter zurück - bis zu den Ideen zur Memex von Vannevar Busch aus dem Jahr 1945.

In diesem Schwerpunktheft zu „CSCW \& Social Computing“ wollen wir den Fokus auf Forschung zu neuen MenschMensch Arbeits- und Netzwerk-Mustern sowie neuen Konfigurationen von Menschen und Computern in Kooperationsszenarien legen. Einreichungen sollten Herausforderungen in der Entwicklung, Einführung und Evaluation von Informationssystemen zur Unterstützung der Zusammenarbeit in Teams, Communitys, Crowds und sozialen Netzwerken in und um Organisationen legen. Nachdem die Unterstützung menschlicher Arbeit durch Informationssysteme sowohl ein Verständnis von technischen Entwicklungen als auch von Arbeitspraktiken benötigt, können und sollten Einreichungen sowohl organisatorische, soziale als auch technische Themen behandeln.

Im Kontext dieses Fokus rufen wir zur Einreichung von Forschungsarbeiten, z. B. zu folgenden Themen auf:

- Generierung von Werten mit Crowdsourcing

- Optimierung von Kernprozessen mit CSCW

- Kooperative Innovationsprozesse: Von der Vision zur Durchführung
- Zukünftige Trends für Kooperationssysteme

- Vertikale Spezialisierung von Kooperationssystemen

- Big Date und Kollaboration

- Organisatorischer Nutzen von Social Media

- Soziale Zusammenarbeit am Digitalen Arbeitsplatz

- Enterprise Social Networks

- Ubiquitäre Kollaboration

- Kooperationssysteme für die Bewältigung von Komplexität

- Kollaboration und Kundenzufriedenheit

- Beobachtete Kollaborationsphänomene und Theorien zur deren Erklärung

\section{Einreichung von Beiträgen}

Bitte reichen Sie Ihre Beiträge bis spätestens 2014-07-01 über das OnlineBegutachtungssystem (http://www. editorialmanager.com/buis/) zur Kategorie „BISE - CSCW \& Social Computing“ ein. Bitte beachten Sie die Hinweise zu formaler Gestaltung und Umfang von Beiträgen für die Business \& Information Systems Engineering (BISE).

Vollständige Beiträge sollten höchstens 50.000 Zeichen einschließlich Leerzeichen umfassen, abzüglich 5.000 Zeichen je Seite an Bildern. Ausführliche Autorenrichtlinien stehen unter http://www. wirtschaftsinformatik.de zum Download bereit.

Einreichungen für das Schwerpunktheft sollen in englischer Sprache erfolgen. Eingereichte Beiträge werden anonymisiert von mehreren Gutachtern in einem doppelt-blinden Verfahren auf Relevanz, Originalität und fachliche Qualität beurteilt. Neben den Herausgebern des Schwerpunktthemas und jenen der Zeitschrift wirken dabei weitere ausgewiesene internationale Persönlichkeiten aus Wissenschaft und Praxis mit. 


\section{Zeitplan}

Einreichung von Beiträgen: 2014-07-01

Benachrichtigung der Autoren:

2014-08-26
Abschluss der ersten Überarbeitung: 2014-10-18

Ggf. Abschluss einer zweiten Überarbeitung: 2015-01-20
Voraussichtliches Veröffentlichungsdatum: Juni 2015 